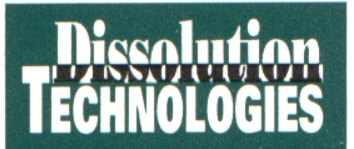

\section{ntroduction}

Pharmaceutical formulations containing more than one component are often analyzed by chromatographic techniques, but multicomponent UV-Vis analysis is now a real alternative. The analysis using UV-Visible spectroscopy is not new, but was traditionally limited by the number of available spectral data and as a consequence by the not very reliable algorithms. Diode-array based instruments and sophisticated algorithms have improved the

\section{Multicomponent Calculation Techniques and Requirements}

The classical method to calculate the individual quantity of the components of a multicomponent mixtures is the so called 'simultaneous equation' method. This method uses only the minimum of spectral information and is sensitive to measurements errors.

\title{
Multicomponent UV-Visible Tablet Dissolution Testing
}

Stephan

Bayerbach,

Ph.D. quality and speed of multicomponent analysis significantly. The following article describes the requirements and techniques to develop a multicomponent analysis method. These will be demonstrated on the example of triamterene and hydrochlorothiazide.
An improvement uses more spectral data or the complete spectrum in the calculation. A so called 'over-determined' system of linear equations is normally solved by least square regression. Effectively, standard spectra are linearly combined to compose a synthetic

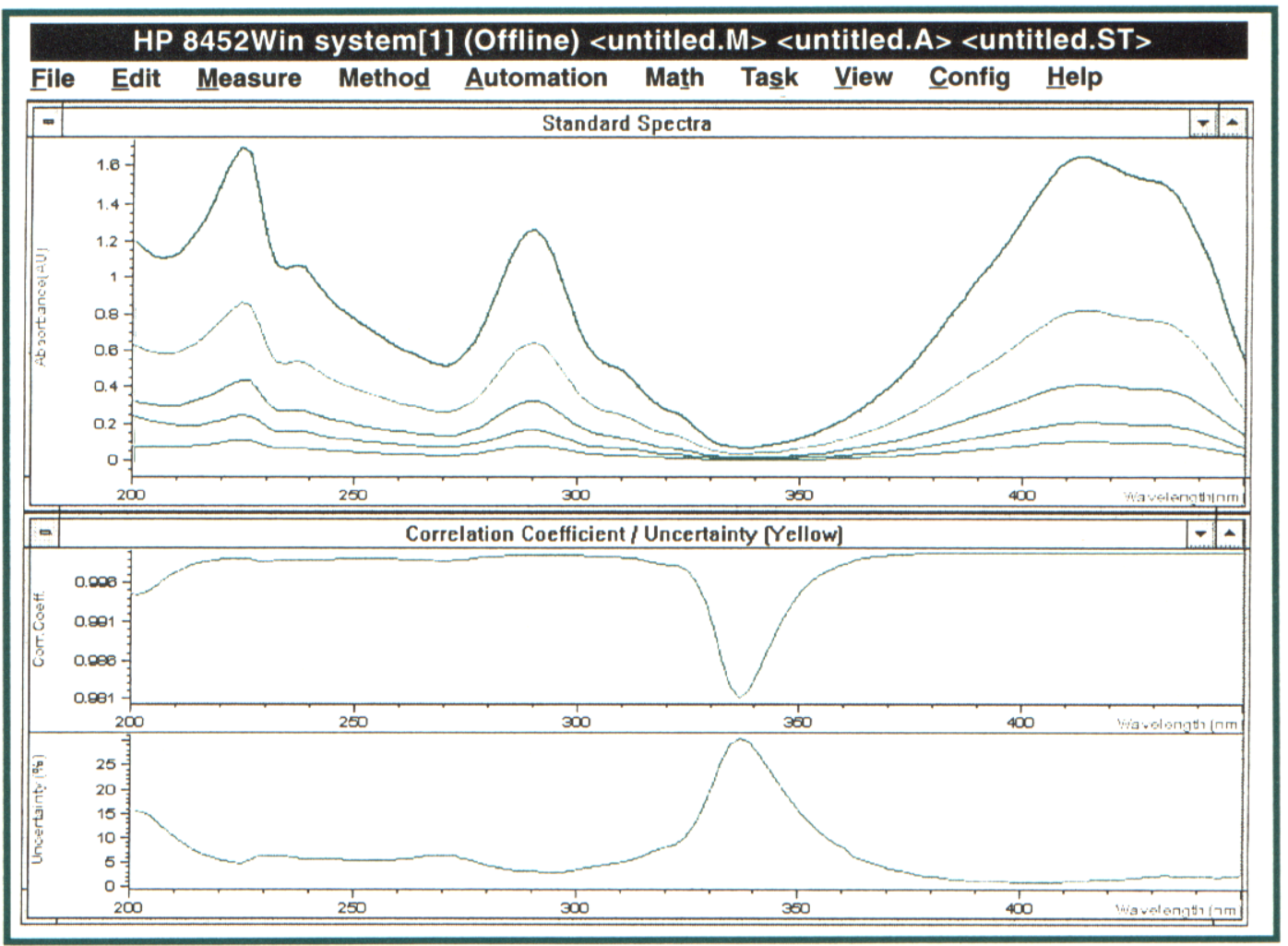

Figure 1: Plot of correlation coefficient/uncertainty versus wavelength. In this example the four best correlation coefficient and the minimum of the uncertainty is at $408 \mathrm{~nm}$, which is not the maximum of the absorbance. 
mixture spectrum as close as possible to the spectrum of the sample under investigation. This is significantly less sensitive to measurement errors of random nature like noise but is still sensitive to the quality of the data, because all data points have equal weight.

A refinement of the least square technique is to use the quality of the data points and use this information to weight the data points in the calculation. Diode-array instruments can measure complete spectra very fast and can

\section{Analysis In}

calculate the standard deviation for each absorbance value.

All the multicomponent quantitative techniques previously described require that:

- There is some degree of spectral difference between the components.

- All components in the mixture, which absorb in the desired wavelength range, must be known and in the calibration set.

- The system obeys Beer's law (linearity).

- There must be no interaction between the components of the mixture that causes spectral changes.

If any one of these assumptions fails then the multicomponent calculation fails.

\section{Developing a Multicomponent Method}

Basically the aim of the development of a multicomponent analysis method is to ensure that the above requirements are fulfilled. In addition, all possible sources of errors which are always inherent in the measurement have to be taken into account.

Some sources for measurement errors can be minimized by optimization of the instrument settings. Noise as a source of random error, can be reduced by time averaging. Drift, as a source of random and systematic error, will cause a baseline shift. This baseline shift between the different measurements can be compensated by
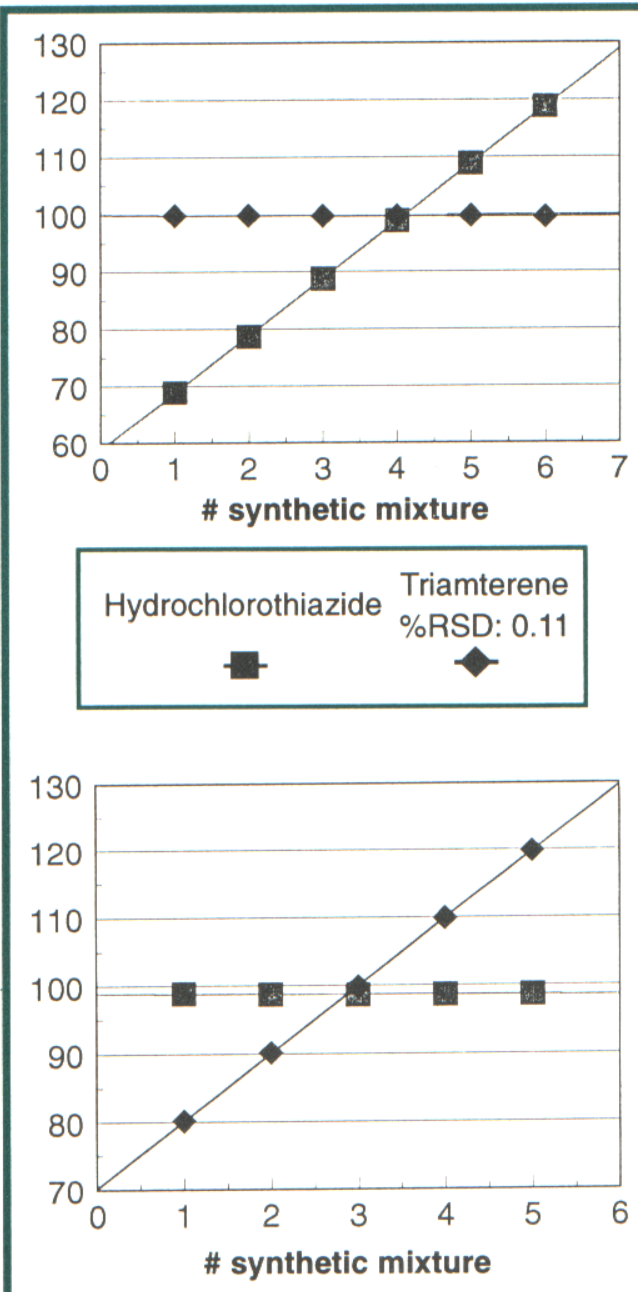

Hydrochlorothiazide \%RSD: 0.09

Triamterene

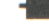

the use of the internal reference technique. Diode-array instruments offer these techniques without the disadvantage of wasting a lot of time for the spectral data acquisition.

The linearity of the system can be influenced by stray light at high absorbance values and concentration dependent deviations from Beer's law due to changes of the extinction coefficients. The reason for these changes may be the formation of dimers at higher concentrations. One way to check the linear relation of absorbance and concentration is to calculate individual linear calibration curves for each component at different concentrations at all wavelengths in a wavelength range. As a measure for the linearity the correlation coeffi-

(continued on page eight)

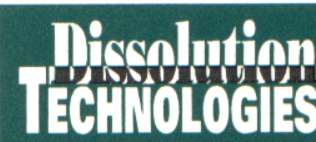

Figure 2: Synthetic mixtures of

triamterene and hydrochlorothiazide. The linearity and reproducibility of these mixtures indicates no problems.

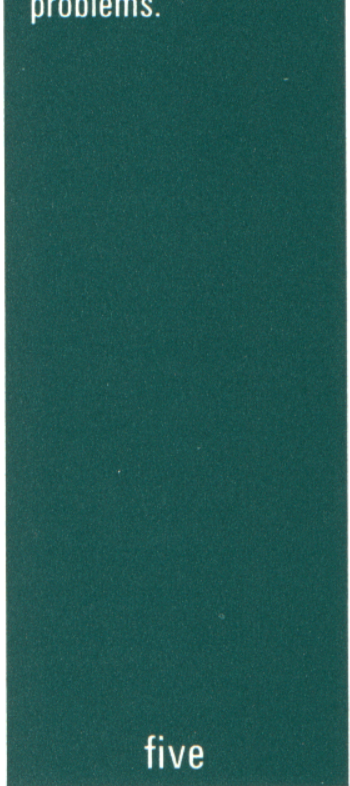




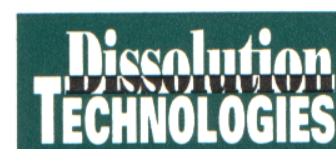

Figure 3: Synthetic mixtures of paracetamol and codeine phosphate. The linearity and reproducibility of codeine phosphate indicates a significant problem.

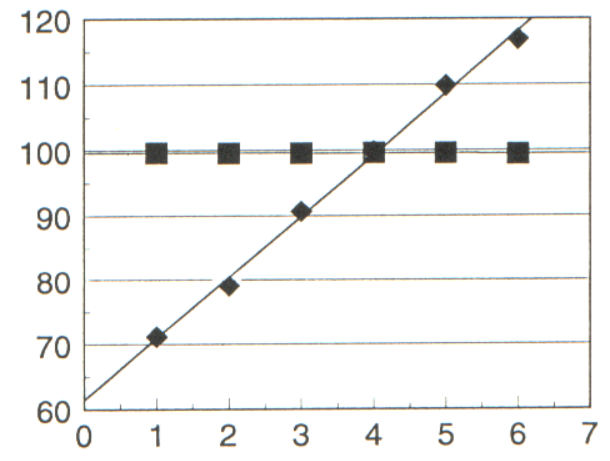

\# synthetic mixtures

\begin{tabular}{|cc|}
\hline Paracetamol & Codeine \\
\%RSD: 0.08 & phosphate \\
& \\
\hline
\end{tabular}

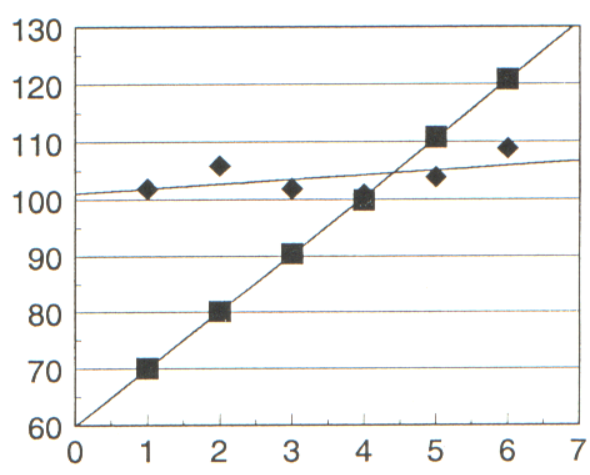

\# synthetic mixtures

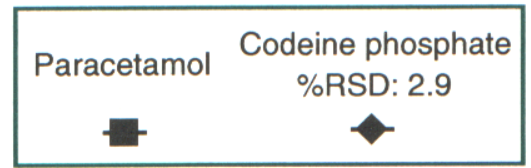

Multicomponent UVVisible Analysis In Tablet Dissolution Testing

eight

\section{(continued from page five)}

cient of a linear regression or the uncertainty can be used. The uncertainty is the deviation of the actual concentration of the standard from the highest concentration and the calculated concentration of the calibration. Figure 1 (see page 4 ) shows a plot of correlation coefficient and uncertainty versus wavelengths calculated from five different concentrations of a yellow dye. The plot allows a fast determination of the ranges where the criteria "linearity" is fulfilled. Based on this information and an inspection of the spectra for wavelength ranges with spectral differences a first selection of the wavelength range to be used for the multicomponent analysis can be made. In some cases the use of the derivatives instead of the absorbance values can improve the spectral differences and suppress baseline shift.

The final selection of the wavelength range for the multicomponent analysis has to be done empirically by analyzing a set of samples. These mixtures have to be prepared from the pure standards, or, preferably, be real samples with known composition. The quality of the results is determined by comparison to the expected values.

A possible experimental design is to prepare for each component a series of synthetic mixtures. In these mixtures the component under investigation is varied between $70 \%$ and $120 \%$ of the target concentration in samples and all other components are held at $100 \%$ of the target. From the results of these tests two pieces of information can be obtained. On the one hand, possible interactions between the components and thereby the linearity of the component which is varied, can be evaluated and, on the other hand, the reproducibility of the component which is held at the same concentration can be calculated. Figure 2 (see page 5) shows the plot and results for a synthetic mixture of triamterene $(50 \mathrm{mg})$ and hydrochlorothiazide $(25 \mathrm{mg})$ in a final volume of about $900 \mathrm{ml}$ dissolution medium. Both, the linearity and reproducibility are good and prove the multicomponent analysis. Figure 3 shows the plot and results for a synthetic mixture of paracetamol $(500 \mathrm{mg})$ and codeine phosphate $(15 \mathrm{mg})$ in a volume of $900 \mathrm{ml}$ dissolution medium and a dilution of 1:3 before the measurement. The component paracetamol can be measured without problems, but codeine phosphate causes problems with linearity and reproducibility. A multicomponent analysis of a mixture of these components with these extreme differences in concentration and absorptivity cannot be done reliably. 
Modern software programs for the calculation of multicomponent analysis also offer statistical tools such as relative fit error, independence of standards and standard deviation which can be used to judge the goodness of fit. Based on all of the above information an analytical method can be implemented and validated.

\section{Experimental}

An HP 8452A spectrophotometer with multicell transport sampling system and HP Vectra PC controller with HP 89550A dissolution software was used. Standards, shown in figure 4 , were dissolved in $0.1 \mathrm{~N}$ hydrochloric acid. Dissolution test on the tablets were done at $37 \mathrm{C}$, a stirring speed of $75 \mathrm{rpm}$, with $900 \mathrm{ml}$ of $0.1 \mathrm{~N}$ hydrochloric acid. The vessels were sampled every 3 minutes for 30 minutes.

\section{Results and Discussion}

Based on initial tests with standard mixtures the analytical conditions shown below were selected. A reference wavelength range was used to minimize the effect of the tablet excipients on the analysis.

Analytical Method:

Analytical wavelength 230 to $400 \mathrm{~nm}$ Reference wavelength 450 to $550 \mathrm{~nm}$ Integration time $1.0 \mathrm{sec}$ Pathlength $1 \mathrm{~mm}$ Data type Absorbance Calculation mode Least square with 'Maximum likelihood'
The results of a dissolution test run using the analytical method are shown in figure 5. The results for both components were very close to the nominal values for the tablets showing no significant interference from the tablet excipient.

\section{REFERENCES}

"The diode-array advantage in UV-Visible spectroscopy", Hewlett-Packard primer, 1988, publication number 12-5954-8912 US pharmacopeia XXII, pp. 1400-1404,1990 "Developing and Validating a Multicomponent UV-Visible Analysis", Hewlett-Packard, 1992, publication number 12-5091-0942E

"Dissolution Testing of a Tablet Formulation of Triamterene and Hydrochlorothiazide", Hewlett-Packard, 1991, publication number 12-5091-1465E

Figure 4: Standard

spectra for

hydrochlorothiazide/

triamterene

formulation.
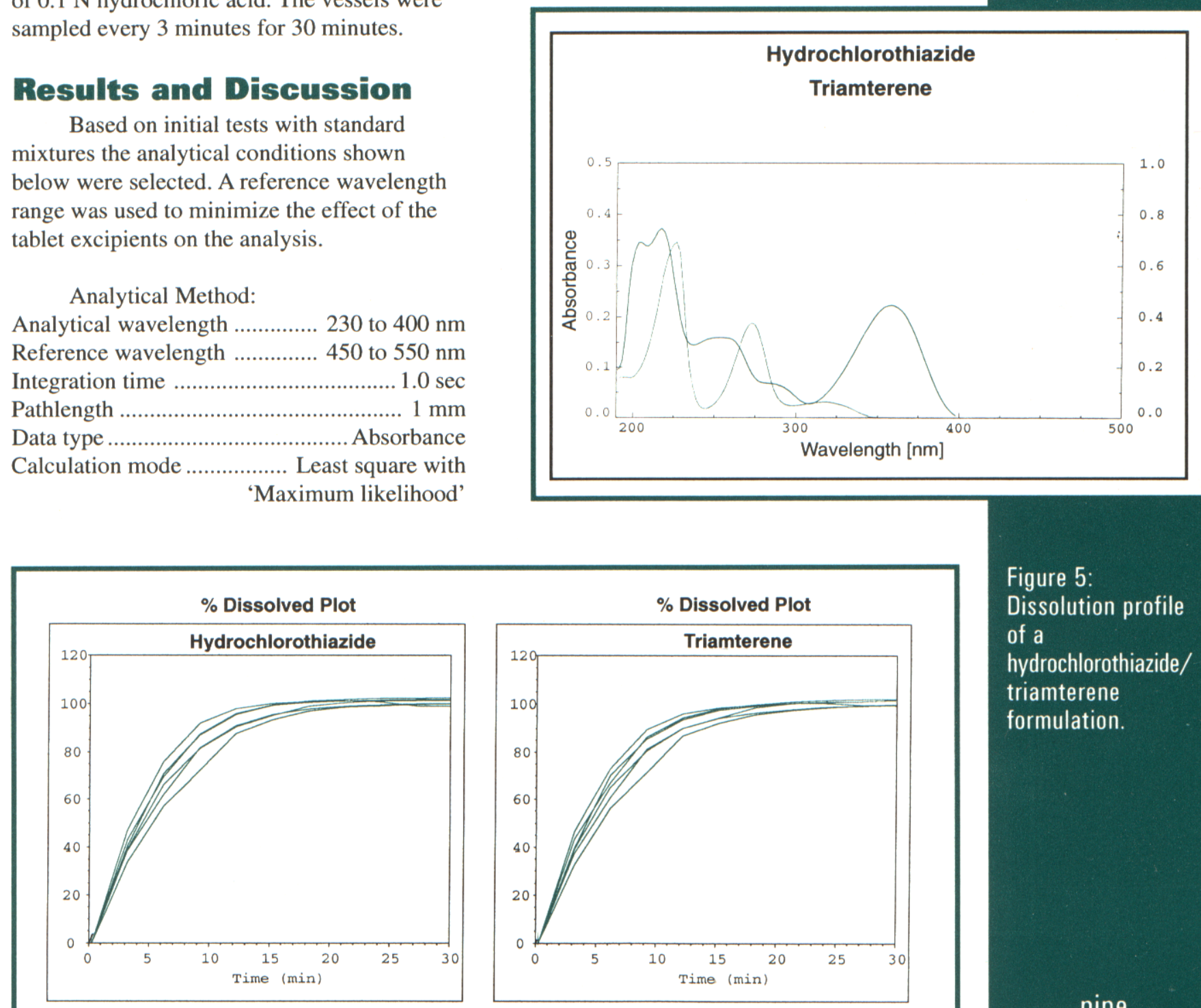

Figure 5:

Dissolution profile

of a

hydrochlorothiazide/

triamterene

formulation. 